\title{
Effect of Color on Animal Behavior
}

\author{
V. V. Egorov* \\ Doctor of chemical Sciences, Professor, Department of chemistry, Moscow state Academy of veterinary \\ medicine and biotechnology, named after K. I. Skryabin. Moscow. Russia
}

*Corresponding Author: V. V. Egorov, Doctor of chemical Sciences, Professor, Department of chemistry, Moscow state Academy of veterinary medicine and biotechnology, named after K. I. Skryabin. Moscow.Russia, Email: kaf_chimii@mgavm.ru

Abstract: The paper discusses the response of higher organisms: dog and cat of color. The correlation of perception of the visible spectrum of organisms with its visual rays was found.

Keywords: The radiation of the eye, the perception of the visible spectrum

\section{INTRODUCTION}

The question of the perception of color by living beings is debated in science for a long time. At the same time, scientists believe that different organisms react primarily to those areas of the visible spectrum that they use in their life. At the present time, there is a certain opinion about the perception of color by a number of higher animals, incl. birds and fish, as well as insects [1]. It is believed that dogs and especially cats, distinguishing about six colors, are poorly oriented in the yellow-green and orange-red regions of the spectrum, but, according to some scientists, should perceive the blue and ultraviolet ranges. Rats (and, apparently, mice) do not distinguish between the blue-yellowgreen area, i.e. oriented only in the orange-red and infrared range.

Above it was a question of perception, associated with the absorption by the visual organs of organisms of light waves of different lengths. No less relevant is their visual radiation, which was proved in $[2,3,5]$. The physicists know Kirchhoff's law: "The electromagnetic frequencies emitted by the body are also absorbed by them". This gives grounds to believe that those spectral ranges that radiate the eyes of organisms can be present in their absorption spectra.

The aim of this work is to study the effect of the visible range of light on the physiological characteristics of animals.

\section{Materials AND Methods}

For work, a number of domestic animals (dogs, cats) were found in the academic vivarium. The experiment was carried out using the RPP method - the reaction of the physiological parameters of the organism [4]. The essence of it is that with the help of an automatic blood pressure monitor (a cuff on the foot of an animal) physiological indicators of the body are measured: systolic and diastolic pressure, $\mathrm{mm}$ $\mathrm{Hg}$, and pulse, $\mathrm{s}^{-1}$, first in the absence (control), then in the presence of a plate painted in a certain color, located 5-10 minutes in front of the animal's eyes at a distance of $10-15 \mathrm{~cm}$.

Comparison of the experimental data with the control data makes it possible to determine in which direction and how much the sum of blood pressure and pulse values ( $\Sigma \Delta$, conditional units) is shifted under the influence of a certain wavelength of light affecting the eyes and thereby evaluate the reaction of the organism to this color.

Spectra of the bioactivity of radiation from the eyes are taken from [5].

\section{RESULTS AND ITS DISCUSSION}

Figure 1 shows the reaction of the cat's physiological parameters to the color of the plate in front of the eyes (curve 2) and the luminescence bioactivity indices $(\Delta \xi)$ corresponding to the emission spectra of his eyes, left and right (curves 1a and 1b) [5]. 


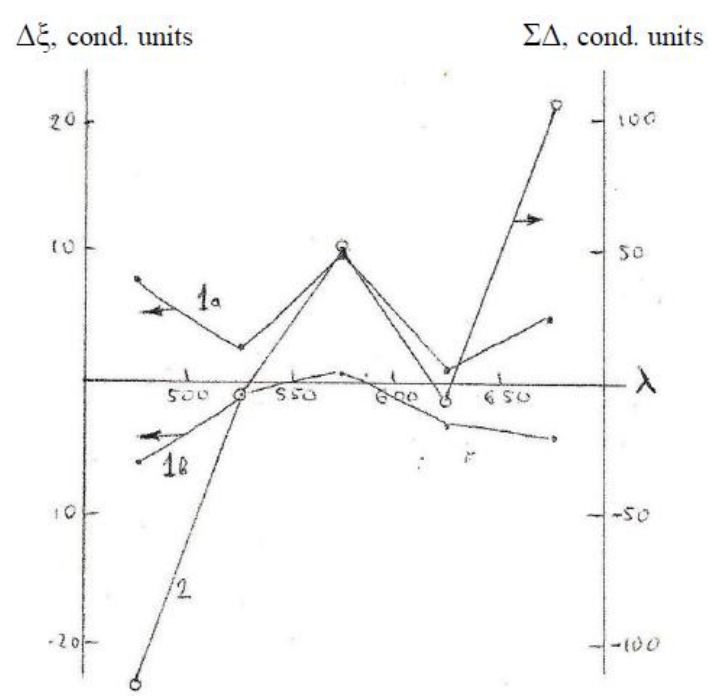

Figure1. Dependence of the sum of the deviations of the physiological parameters of the cat under the influence of color (curve 2, the method of RPP, 5 min., $10 \mathrm{~cm}$ ) and the luminescence index of its left (curve 1a) and right eye (curve 1b, method "indicators of germination of seeds" [6], $10 \mathrm{~min}, 1$ m) from the wavelength, $\mathrm{nm}$ (abscess). $20+0.2^{\circ} \mathrm{C}$. See explanation in the text.

As can be seen in the figure (curve 2), the most active reaction of the animal is observed in the blue and red regions of the spectrum and quite distinct in yellow, while in the green and orange regions it is practically absent. In the last two regions, judging by the bioactivity index of the luminescence of the eyes (curves la and $\mathrm{lb}$ ), weak visual radiation is observed. However, judging by the graphs, it is quite distinct in the blue and red regions of the spectrum and quite definite in yellow (with a positive index for both eyes).

A comparison of the data of the two experiments shows that the spectral regions of the glow of the cat's eyes correspond to the colors of its active reaction, which is obviously associated with their absorption. At the same time, the analysis of literature data (see above) shows that a poor orientation in the green (confirmed by our experiments with colored food) and orange regions of the spectrum corresponds to a weak glow in the eyes of the animal in these ranges (see Fig. 1). At the same time, as noted above, in the yellow and red regions, as well as in the blue, its reaction, judging by the change in physiological parameters, is quite active. Thus, we see the correspondence of the spectra of the glow of the eyes of a given cat to the spectra of its activity.

Figure 2 shows the data for two postoperative dogs of small breeds of 4 and 5 years. Here, curve 1 corresponds to the index of activity of the visual radiation of one animal (method [6]), and curve 2 - to the change in the amount of physiological indices of the other one under the influence of plates of different colors (RPP method). In the case of the first dog the radiation of the eyes in the blue and orange regions of the spectrum and its practical absence in the red, yellow and green ranges is noticeable. In the same ranges in the second animal, we see a negative reaction of physiological parameters, which is practically on the same level for all three colors.

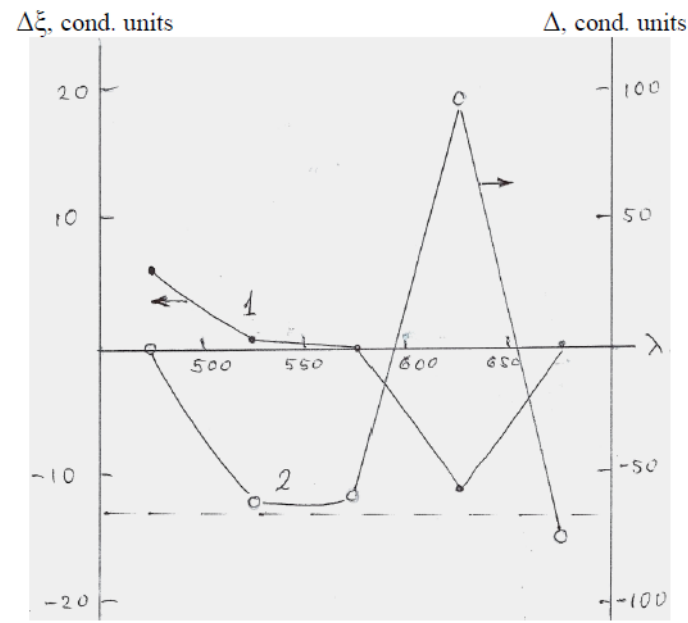

Figure2. The dependence of the sum of the deviations of the physiological parameters of the first dog under the influence of color (curve 2, the method of "reaction of physiological indices", $5 \mathrm{~min}$., $10 \mathrm{~cm}$ ) and the index of bioactivity of the luminescence of the right eye of the second dog (curve 1, method "indicators of germination of seeds" [6], $10 \mathrm{~min}, 1$ m) from the wavelength, $\mathrm{nm}$ (abscess). $20+0.2^{\circ} \mathrm{C}$. See explanation in the text.

A comparison of the two curves suggests that this lowered level in the second dog (see the dashed curve in Figure 2) is the reference level. Its decrease may be due to the suppression of the activity of the animal kept by an extraneous person (which was not observed in the case of cat with the owner held - Fig. 1), in a confined space bounded by a colored plate. In this case, deviations from it can be considered as a reaction of the organism to the spectral regions. They are observed, as seen in Fig. 2 (curve 2), in the blue and orange regions of the spectrum, which corresponds to the spectrum of the glow of the eyes of the first animal (curve 1). This allows us to relate the spectra of the glow of the dogs' eyes to their reaction to colors. The comparison of the data obtained with the literature shows that the yellow-green and red areas, marked as passive for the perception of the animal, really correspond to its weak physiological reaction (Figure 2, dotted line for curve 2) and low glow of the eyes (points on the 
abscissa of the curve 1), while in the orange area both are quite noticeable.

Thus, the data obtained by us and analysis of the literature allow us to conclude that the higher the emissivity of the eye of the organism in a given region of the spectrum, the more noticeably this region affects its physiology and behavior, apparently primarily as a result of its active absorption by the visual organ.

\section{REFERENCES}

[1] Skyjournal.ru/mir-glazami-zhivotnih-67

[2] Egorov V. V. Glow eyes - fact or fiction?/ /Nanotechnology and health. 2012. №1. P. 10 16.
[3] Egorov V. V., Tsetlin V. V. To the problem of radiation of eyes.//Veterinary medicine, Zootechny. Biotechnology. 2016. № 8. P. 82 84.

[4] Egorov V. V. the Relationship of biological activity of water with the intensity of the geomagnetic field.//In proceedings of "Issue 72". ZooVetBook. 2017. C. 16.

[5] Egorov V. V. Spectrum the radiation of the eye.// Veterinary medicine, Zootechny, Biotechnology. 2017. № 5. P. 107 - 111.

[6] RF patent 2325842.

Citation: V. V. Egorov. Effect of Color on Animal Behavior. ARC Journal of Animal and Veterinary Sciences. 2017; 3(3):8-10. doi: dx.doi.org/ 10.20431/2455-2518.0303002.

Copyright: (1) 2017 Authors. This is an open-access article distributed under the terms of the Creative Commons Attribution License, which permits unrestricted use, distribution, and reproduction in any medium, provided the original author and source are credited. 